\title{
A Summary of Content-Based Image Retrieval Methods
}

\author{
Zhongmin $\mathrm{Li}^{1, \text { a }}$ and Haochen $\mathrm{Wu}^{2, \mathrm{~b}}$ \\ ${ }^{1}$ School of Information Engineering, Nanchang Hangkong University, Nanchang, China \\ ${ }^{2}$ School of Information Engineering, Nanchang Hangkong University, Nanchang, China \\ a zhongmli@163.com, b464417131@qq.com
}

Keywords: CBIR; Feature extraction; semantic gap; relevance feedback

Abstract. This paper mainly focuses on the content-based image search system. First introduces the research status of CBIR in China and abroad, and then proposes and illustrates two key problems affecting the accuracy of the system, summarizes commonly used methods to solve these two problems.

\section{Introduction}

With the rapid development of Internet, massive data and information are closely related to people's life. Image, video and other multimedia information is rapidly increasing, How to find the necessary information accurately and efficiently from the massive information database is a hot issue in the information era. Traditional search engine use text as search object, realize information search through the keyword, text-based search technology has been very mature. For example, Google, Baidu and other engines have provided a convenient text search. However, the flaw of the text search is that it is impossible to retrieve pictures which is difficult to describe in words, and it is different to express people's search intention directly and comprehensively by words. Content-based image retrieval technology can be a good solution to this problem.

Content-based image retrieval realize information search by uploading images instead of text, computer extract the characteristics of the image automatically, then find out the similar images from the image library, that is, search for similar images in the image library by uploading images, use a image as search handle compatible with the visual habits of people. It can be able to express the search intention directly, and it can also search for pictures that are difficult to describe with words. Therefore, content-based image retrieval has become a hot spot in the current search.

\section{Research Status}

Content-based image retrieval emerged from 1990s, after more than and 20 years of research, there are some great progress in algorithm optimization and practical application.

In foreign countries, some agencies have introduced the content based image retrieval system. Including the image retrieval system based on global image features, matching similar images by extract color ,texture and other images low-level features, such as: QBIC system developed by IBM is the first commercial content-based image retrieval system, VIR[1] image search system developed by Virage, VisualSEEK[2] and WebSEEK developed by Columbia University and Photobook[3] system whose face detection technology has been adopted by the police station; Image retrieval system based on local features, extracting features of regions of interest in images by image segmentation, Such as: Netra[4] image retrieval system developed at the University of California, Blobworld[5] image retrieval system research by Berkeley University, PicToSeek[6] image retrieval system developed by the computer science department in University of Amsterdam; Image retrieval system with relevance feedback, reduce the semantic gap by user feedback or machine learning, such as: MARS[7] multimedia analysis and retrieval system.

In China, the complete CBIR system is not many, the research of CBIR is concentrated in the University and some scientific research institutions, at present mainly in the theoretical research 
stage.

\section{Key Problems}

The content-based image retrieval algorithm mainly has two key problems, which are accurate and comprehensive extraction of image features and reduce the semantic gap.

Feature Extraction. Each image has different features, in the image retrieval algorithm, we need to compare and judge the similarity between images according to the different features of the image, so as to find the relevant image. Therefore, comprehensive and accurate extraction of image features directly affect the precision ration of content-based image retrieval system, feature extraction algorithm is one of the most important part of CBIR system. The following are introductions of several hot spots in feature extraction at present.

Image Segmentation. Image segmentation is an important processing step in the accurate extraction of image features, especially in shape feature extraction; we can extract the features of the specific region of the image and the relationship between the regions through image segmentation. Because of the complexity and variety of the images, image segmentation has always been a difficult problem and a hot spot in the field of image processing. At present, the popular image segmentation algorithm has several categories: Thresholding Method, Edge Detection Method, Region-growing Method, Cluster Analysis Method and Particle Swarm Optimization and Genetic Algorithm. In Edge Detection Method, the operators commonly used including Canny operator [9], LoG operator [10] and Sobel operator [11], etc. Commonly used clustering methods include K-means clustering [13], fuzzy C-means clustering [14]. Commonly used Particle Swarm Optimization and Genetic Algorithm include Ant Colony Optimization[15], Artificial Bee Colony Algorithm[16] and Cuckoo Search[17] etc.

Basic Features. Classic image features include the most basic features match with human visual perception, color features, texture features and shape features are commonly used. These features conform to people's visual habits, their extraction algorithm has been studied and developed from the beginning of image retrieval. Therefore, extraction algorithms of these features are relatively mature. At present, the main methods of extracting color feature[18] include color histogram, color moment, color aggregation vector, color correlogram, Texture feature extraction methods mainly include [19-22]: Gray level co-occurrence matrix, Local binary patterns, Tamura texture features and wavelet transform method; The main features of the shape feature extraction methods include[23-25]: Fourier shape description method, Shape invariant moment, Boundary feature description method.

Other popular feature extraction algorithm. In addition to the basic visual features of the image, there are a number of good features and algorithms are often used, and then introduce two kinds of these.

SIFT feature extraction algorithm[26]: SIFT is scale invariant feature transform, it is used to extract the local features of the image. It finds the feature points in the scale space and finds its' location, scale and rotation invariants. It has scaling, rotation, affine invariance, so, it can get better image recognition results.

Hashing Algorithms[27]: Mapping the image to the binary space which is low dimension, using the hash encoding to represent the image feature. It greatly reduces the data dimension, approximate nearest neighbor search algorithms, it can greatly increase the efficiency of retrieval and reduce data storage space.

Semantic Gap. In content-based image retrieval system, computer matches the two images by extracting the features of images. However, in fact, people determine the similarity of two images is not only to rely on the low-level visual features of the image; people's understanding of the image depends on the common sense and experience. Simple image visual feature semantics become different from image semantics people understand in fact .This difference called the semantic gap. In content-based image retrieval system, the final result is relying on the semantics people understand, so, to reduce and eliminate the semantic gap is very important. 
Relevance feedback is the main method to reduce the semantic gap in current image retrieval system. At present, the commonly used relevance feedback algorithms are the following:

Relevance feedback algorithm based on feature weight adjustment[28-29]: By analyzing the semantic information from user's feedback, adjust the weights of relevant features in the system, increase the feature weight which user consider it has large correlation degree, and reduce the feature weight which user consider it has little correlation degree, then matching images according to the feature vector after adjustment.

Relevance feedback algorithm based on machine learning[30-33]: This algorithm is regard the image retrieval problem as a classification problem, divide the retrieval results into related and unrelated, regard the process of user's interaction as a training process, understand the user 's semantics gradually and update the search results. The most commonly used machine learning theory is SVM (support vector machine),In addition there are neural networks, genetic algorithms, particle swarm optimization algorithm, etc.

\section{Conclusions}

In recent years, content-based image retrieval technology has been developing rapidly. Some companies have launched image search engines, such as Google and Baidu, these search engine can achieve a basic search function, but the results are always unsatisfactory, key problem is that the image feature extraction and semantic gap, with the rapid development of the computer field, some of the optimization algorithms used in the image search field have got great progress.

This paper summarizes the research situation of content-based image retrieval in last ten years, lists some popular methods and algorithms, and illustrates two important factors which impact the accuracy of CBIR system, feature extraction and semantic gap, the content-based image retrieval system will development towards optimizing and solving the two key problems. This paper presents some of the most commonly used solutions, and hope to have a reference for future research.

\section{Acknowledgments}

This work was financially supported by the Programs of the National Natural Science Foundation of China (Grant No. 61263040).

\section{References}

[1] J. R. Bach, C. Fuller, A. Gupta, et al: SPIE, Vol. 2670 (1996), p.76-87

[2] J. R. Smith, and S. F. Chang: Proc. ACM, (1997), p.87-98

[3] A. Pentland, R.W. Picard, and S. Sclaroff; Proc. SPIE, Vol. 2185(1994), p.34-47

[4] W. Y. Ma, and B. S. Manjunath: Proc. IEEE, (1997), p.925-928

[5] C. Carson, M. Thomas, S. Belongie et al: Proc. Visual Information Systems, Vol. 6(1999), p.509-516

[6] T. Gevers, and A. Smeulders: Proc. VISUAL97, (1997), p.93-100

[7] W. Y. Ma, and B. S. Manjunath:. Proc. IEEE, (1997), p.925-928

[8] L. Jianwu: Research on Key Techniques of Image Thresholding. Jilin University, (2014)

[9] Z. Fenglin: Research on Contour-based Image Matching Technology. Nanjing University of Aeronautics and Astronautics, (2010)

[10] H. Yue: Research on Gray-level Image Segmentation in Complex Background. Taiyuan University of Technology, (2008) 
[11] Z. Hui, and G. Shenrong: Computer Science, Vol. 38(2011), p.278-280

[12] L. Zhengguo: The Segmentation and Realization of High Spatial Resolution Remote Sensing Image Based on Region Growing Algorithm. Guangxi University, (2008)

[13] T. Zhengjie: Research on Road Segmentation and Extraction's Algorithms of High-resolution Remote Sensing Images. Xi'an University of Science and Technology, (2009)

[14] Z. Gui: Image Segmentation Algorithm Based on Fuzzy C-menas. Xiangtan University, (2013)

[15] C. Xiaomei: Research on Image Segmentation Method Based on Ant Colony Algorithm. University of Electronic Science and technology, (2011)

[16] L.Jianhui: A New Intelligent Optimization Algorithm and Its Application in Image Segmentation. Shaanxi Normal University,(2011)

[17] L. Xinni: Computer Engineering, Vol. 39(2013), p.274-278

[18] W. Yi: Research on Image Search Based on Color. University of Electronic Science and technology, (2011)

[19] M. Benco, R. Hudec: R adioengineering, Vol. 4(2007 ), p.64-67

[20] L. Nanni, S. Brahnam, and A. Lumini: Expert Systems with Applications, Vol. 37(2010), p.7888-7894

[21] H. Tamura, S. Mori, and T. Yamawaki: IEEE Trans On Systems, Man and Cybernetics, Vol. 8(1978), p.460-473

[22] H. Deng-sheng, A. Wong, M. Indrawan, etal: Proc. IEEE, (2000), p.392-395

[23] W. Wei, F. Xuezhi, and P. Xiao: Journal of Remote Sensing, Vol. 15(2011), p.73-87

[24] GUO Erjun: An Image Retrieval Algorithm Based on Shape Feature Matrix. Xinyang Normal University, (2014)

[25] A. Khotanzad, Y. H. Hong: IEEE Transactions on Pattern Analysis \& Machine Intelligence, Vol. 12(1990), p.489-497

[26] L. Zhang , J. Chen , Y. Lu , etc: ICYCS 2008, (2008), p.1766-1770

[27] F. Haiyan: Research on Hashing-Based Image Retrieval for Large-Scale Dataset. Dalian University of Technology, (2014)

[28] P. Qirong: Research on Content-based Image Retrieval and Recommendation Technology. Northwestern University, (2015)

[29] Z. Xiao: Study on the Image Retrieval Technology Based on Shape Features and Relevance Feedback Mechanism. Beijing Institute of Graphic Communication, (2015)

[30] C. Ma, Q. Dai , J. Liu , etc: International Journal of Digital Earth, Vol. 7(2014), p.725-745

[31] Jun-Hua Han, De-Shuang Huang, Tat-Ming Lok, et al: Vol. 4(2005), p.2561 - 2564

[32] G. Yuan, G. Guohua, and L. Xiaonin: Journal of Northwestern University(Natural Science edition), Vol. 35(2005), 379-382

[33] T. Zhaoxia, Z. Hui, X. Dongmei: Scientific Journal of Computer Science, Vol. 38(2011), p.278-280 